\title{
Infectious Diarrhoea with SIRS from Yokenella regensburgei
}

Milori $\mathrm{A}^{1 *}$, Miliori $\mathrm{E}^{2}$, Gkatsis $\mathrm{I}^{3}$, Koletti GB ${ }^{3}$, Vogiatzi $\mathrm{A}^{3}$, and Paulou $\mathrm{A}^{1}$

${ }^{1}$ Department of Microbiology, Hatzikosta Hospital Messolonghi, Greece

${ }^{2}$ School of Medicine National and Kapodistrian University of Athens, Greece

${ }^{3}$ Department of Internal Medicine, Hatzikosta Hospital Messolonghi, Greece

\section{Background}

Yokenella regensburgei is an opportunistic human pathogen, a gramnegative, oxidase negative, motile rod, fermentative bacterium whose infections are rarely reported in humans [1]. Only 6 cases have been previously reported regarding septic knee [2], urinary tract infection [3], transient bacteraemia [2], perimalleolar ulcer [4], cellulitis [5], septic shock [6] and enteric fever [7] similar to the present case.

It belongs to the family Enterobacteriaceae [8] and it is biochemically similar to the bacterium Hafnia Alvei with their difference being that $H$. alvei is resistant to colistin and VP negative whereas Y. regensburgei has the opposite characteristics [9]. The National Institutes of Health in Japan identified it as NIH biogroup 9 and the Centers of Disease Control and Prevention as enteric group 45, proposing the name Koserella trabulsii. The name Yokenella regensburgei proposed by Kosako et al. finally prevailed over $K$. trabulsii $[1,2]$. The bacterium has been isolated from intestinal tracks of insects and reptiles, well water and salad [7] as well as from many human anatomical sites including blood [6], faecal samples, upper respiratory tract, urine [2,3] and knee fluid $[9,10]$. The small number of the cases reported with Y. regensburgei as a pathogen is responsible for the shortage of epidemiological and clinical information.

Here we present a case from Y. regensburgei which is to our current knowledge the first case reported in Greece.

\section{Case Report}

A 17-year old male was transported to General Hospital of Messolonghi from the community Health Centre of Astakos with 6-7 daily episodes of diarrhea, fever up to $39.8^{\circ} \mathrm{C}$ and chills. The patient reported that these smptoms emerged three days before his arrival to the Health Centre of Astakos, three hours after physical exercise and that the fever would not subside with the consumption of paracetamol and mefenamic acid. He arrived to General Hospital of Messolonghi on the fourth day of the symptoms.

Physical examination with deep palpation of abdomen showed sensitivity in the entire abdominal wall and increased bowel sounds. A systemic examination revealed nothing of note. Patient's medical history showed only cefuroxime allergy. His vital signs were: blood pressure $135 / 60 \mathrm{~mm} / \mathrm{Hg}$, pulse rate $105 / \mathrm{min}, \mathrm{SO}_{2} 98 \%$, body temperature $39.0^{\circ} \mathrm{C}$. Upper and lower abdominal sonography results were normal. Laboratory examination showed leukocytosis (WBC $\left.13270 / \mathrm{mml}^{-3}\right)$, neutrophilia $(76.7 \%$, normal range $40 \%$ to $74 \%)$, absolut neutrophil count $10180 / \mathrm{mml}^{-3}$, lymphocytes $11.8 \%$ (19\% to $48 \%$ ), monocytes $7 \%$ (3.4\% to $9 \%$ ), eosinophils $1.5 \%$ ( $0 \%$ to $7 \%$ ), increased creactive protein (CRP) $18.76 \mathrm{mg} / \mathrm{dl}(0 \mathrm{mg} / \mathrm{dl}$ to $0.8 \mathrm{mg} / \mathrm{dl})$, hemoglobin $14.2 \mathrm{mg} \mathrm{dl}^{-1}$ and MCV $78.5 \mathrm{fL}$. The rest of the biochemical test results were normal. Widal test for typhoid and Wrghit tests were negative. Blood smear examination showed neutrophils with toxic granulation and WBC differential showed band neutrophils $12 \%$, neutrophils $67 \%$, lymphocytes $12 \%$, monocytes $7 \%$, eosinophils $2 \%$. General stool examination showed a large amount of white blood cells, erythrocytes and mucous. Parasitological examinations' results were negative.
Two stool culture samples were prepared in two days and after a 48-hour incubation, bacteria growth was observed on SS agar in both samples. This bacterium was non lactose fermenting with opaque colony morphology unlike the transparent colonies of shigella. Biochemical testing of the microorganism showed that the bacterium was weakly positive for catalase and negative for oxidase, urease, indole and $\mathrm{H}_{2} \mathrm{~S}$. The identification testings were performed by VITEK 2 Compact automated system (Bio Merieux, France) and identified the bacterium as Yokenella regensburgei. Biochemical tests of Vitek-2 are presented in Table 1 .

The stool cultures were negative for the following Enteropathogens: Salmonella, Shigella, Campylobacter, Enterohemorrhagic E. coli, Y. enterocolitica.

Antimicrobial susceptibility test was performed using Disk diffusion: Mueller-Hinton agar (MHA) method according to the clinical and laboratory standards institute (CLSI) guidelines (Zone Diameter and minimal inhibitory concentration (MIC) Interpretive Standards for Enterobacteriaceae) [11]. The microorganism was resistant to ampicillin and colistin, intermediate to amoxicillin/clavulanic acid and sensitive to Cefotaxime, Amikacin, Gentamicin, Cefuroxime, Cefixime, Ciprofloxacin, Imipenem, Meropnem, Aztreonam and Trimethoprim/ Sulfamethoxazole.

The patient received treatment with cefixime and his symptoms were resolved. After 3 days of hospitalization he was dismissed and continued the treatment with cefixime for six more days and was recommended a follow up 10 days after the end of the treatment. Clinical examination showed no evidence of relapse.

\section{Conclusion}

The track of the pathogen transmission remains unclear [6,7]. The patient of this case reported that three hours before the emerge of the symptoms (abdominal pain and diarrhea) he had returned home after his sport activity, boiled an egg and consumed it. There appears no correlation between this incident and the other cases reported [2-7]. Although in five of the six previous cases the patients were immune suppressed (alcohol consumption, adenocarcinoma, chronic renal failure, diabetes mellitus and use of steroids were reported) [2-6], the patient of the present case had no such medical history and seemed to be immunocompetent. The previous cases involved patients of an older

*Corresponding authors: Ariana Milori, Department of Microbiology Hatzikosta Hospital Messolonghi, Greece, Tel: 00306983913128; E-mail: arianamilori@gmail.com

Received January 04, 2017; Accepted June 07, 2017; Published June 09, 2017

Citation: Milori A, Miliori E, Gkatsis I, Koletti GB, Vogiatzi A, et al. (2017) Infectious Diarrhoea with SIRS from Yokenella regensburgei. J Med Microb Diagn 6: 255. doi:10.4172/2161-0703.1000255

Copyright: @ 2017 Milori A, et al. This is an open-access article distributed under the terms of the Creative Commons Attribution License, which permits unrestricted use, distribution, and reproduction in any medium, provided the original author and source are credited. 


\begin{tabular}{|c|c|c|c|c|c|c|c|c|c|c|c|c|c|c|c|c|c|}
\hline \multicolumn{18}{|c|}{ Biochemical Details } \\
\hline 2 & APPA & - & 3 & ADO & - & 4 & PyrA & - & 5 & IARL & - & 7 & dCEL & - & 9 & BGAL & + \\
\hline 10 & $\mathrm{H} 2 \mathrm{~S}$ & - & 11 & BNAG & + & 12 & AGLTp & - & 13 & dGLU & + & 14 & GGT & - & 15 & OFF & + \\
\hline 17 & BGLU & + & 18 & dMAL & + & 19 & dMAN & + & 20 & dMNE & + & 21 & BXYL & - & 22 & BAlap & - \\
\hline 23 & ProA & - & 26 & LIP & - & 27 & PLE & - & 29 & TyrA & + & 31 & URE & - & 32 & dSOR & - \\
\hline 33 & SAC & - & 34 & dTAG & - & 35 & dTRE & + & 36 & $\mathrm{CIT}$ & - & 37 & MNT & - & 39 & $5 K G$ & - \\
\hline 40 & ILATk & + & 41 & AGLU & - & 42 & SUCT & + & 43 & NAGA & - & 44 & AGAL & + & 45 & PHOS & + \\
\hline 46 & GlyA & - & 47 & ODC & + & 48 & LDC & + & 53 & IHISa & - & 56 & CMT & + & 58 & O129R & + \\
\hline 59 & GGAA & - & 61 & IMLTa & - & 62 & ELLM & - & 64 & ILATa & - & & & & & & \\
\hline
\end{tabular}

Table 1: Biochemical test results of Vitek-2 Compact automated system for Y. regensburgei.

age [2-6]. Only one case has been reported of a young person (aged 5) who was immunocompetent previous to the present [7].

The patient met the SIRS (Systemic Inflammatory Response Syndrome) criteria which involve two or more of the following: body temperature over $38^{\circ} \mathrm{C}$ or under $36^{\circ} \mathrm{C}$, pulse rate over $90 / \mathrm{min}$, respiratory rate over 20 breaths $/ \mathrm{min}$ or $\mathrm{PaCO}_{2}$ under $32 \mathrm{mmHg}$, WBC over 12000 or less than $4000 \mathrm{mml}^{-3}$ [12]. The patient showed body temperature $39.8^{\circ} \mathrm{C}$, pulse rate $105 / \mathrm{min}$ and $\mathrm{WBC} 13270 \mathrm{mml}^{-3}$.

Yokenella regensburgei is a potentially dangerous pathogen that has been rarely isolated by humans [7]. It is highly possible that some cases of $Y$. regensburgei have not been identified, as the stool culture results show opaque colony morphology on SS agar, which does not indicate a common enteropathogen colony and therefore we do not continue to the identification process.

\section{References}

1. Kosako Y, Sakazaki R, Yoshizaki (1984) Yokenella regensburgei gen. nov., sp. nov: A new genus and species in the family Enterobacteriaceae. Jpn J Med Sci Biol 37:117-124.

2. Abbott SL, Janda JM (1994) Isolation of Yokenella regensburgei ("Koserella trabulsii") from a patient with transient bacteremia and from a patient with a septic knee. J Clin Microbiol 32: 2854-2855.

3. Aziz Z (2015) Emerging of Yokenella regensburgei as uropathogen: First Report. J Biol Agric Healthc 5.
4. Olivares MF, Palenciano JB, Laffón IM, Ruiz León JM (2005) Yokenella regensburgei infection in a perimalleolar ulcer. Med Clin (Barc) 125:358-359.

5. Bhowmick T, Weinstein MP (2013) A deceptive case of cellulitis caused by a gram-negative pathogen. J Clin Microbiol 51: 1320-1323.

6. Fill M, Stephens J (2009) Abdominal abscess and septic shock secondary to Yokenella regensburgei. Inter J Infec Dis 9.

7. Jain S, Gaind R, Gupta KB, Dawar R, Kumar D, et al (2013) Yokenella regensburgei infection in India mimicking enteric fever. $\mathrm{J}$ Med Microbiol 62: 935-939.

8. Stock I, Sherwood KJ, Wiedemann B (2004) Antimicrobial susceptibility patterns, (beta)-lactamases, and biochemical identification of Yokenella regensburgei strains. Diagn Microbiol Infect Dis 48: 5-15.

9. Kosako Y, Sakazaki R, Huntley-Carter GP, Farmer JJ (1987) Yokenella regensburgei and Koserella trabulsii are subjective synonyms. Int $\mathrm{J}$ Syst Bacteriol 37:127-129.

10. Hickman-Brenner FW, Huntley-Carter GP, Fanning GR, Brenner DJ, Farmer JJ (1985) Koserella trabulsii, a new genus and species of Enterobacteriaceae formerly known as Enteric Group 45. J Clin Microbiol 21:39-42.

11. CLSI (2014) Performance standards for antimicrobial susceptibility testing; 22nd Informational Supplement M100-S22. Wayne, PA: Clinical and Laboratory Standards Institute.

12. Singer M, Deutschman CS, Seymour CW, Hari MS, Annane D, et al. (2016) The third international consensus definitions for sepsis and septic shock (Sepsis-3). JAMA 315:801-810. 